\title{
Análisis de la Industria 4.0 como factor diferenciador del Sector Industrial del Ecuador
}

\author{
Oswaldo Wilfrido Alvarez Vásquez \\ owalvarez@uce.edu.ec \\ Flavio Roberto Arroyo Morocho \\ frarroyo@uce.edu.ec \\ Universidad Central del Ecuador - Instituto de Postgrado \\ Facultad de Ingeniería y Ciencias Aplicadas \\ Quito - Ecuador
}

\section{RESUMEN}

La implementación de la industria 4.0 a nivel mundial avanza a diferente velocidad que las industrias emergentes, la migración a la industria inteligente se realiza con las restricciones de la infraestructura existente y capacidad tecnológica instalada, de aquí parte la brecha que complica la adopción de la industria 4.0. Específicamente, en Ecuador todavía no es visible una política pública o el interés privado para dar ese salto a la nueva revolución industrial. El presente trabajo plantea una metodología que permita, en primera instancia evaluar de manera cualitativa y rápida cual es el estado actual del nivel de madurez de la implementación de la industria 4.0 en función de factores de éxito que son resultado de la experiencia de países que ya han generado lecciones aprendidas en dicha implementación, y resulta más sencillo enfocar los esfuerzos para sentar las bases de la cuarta revolución industrial, identificar el estado actual de la industria para direccionar las políticas públicas, la inversión privada. Para identificar la situación actual de la industria 4.0 en Ecuador, se propone evaluar el nivel de madurez de acuerdo con los siguientes cinco factores de éxito: estrategia, tecnología, capacidad de innovación, ecosistemas de innovación y manejo del cambio.

Palabras clave: Industria 4.0; Estrategia; Innovación; Manejo Del Cambio 


\title{
Industry 4.0 analysis as a key factor for Industrial Sector of Ecuador
}

\begin{abstract}
The global implementation of industry 4.0 is advancing at a different speed than emerging industries, the migration to smart industry is carried out with the restrictions of the existing infrastructure and installed technological capacity, hence the gap that complicates the adoption of the industry 4.0. Specifically, in Ecuador a public policy or private interest is not yet visible to make that leap to the new industrial revolution. The present work proposes a methodology that allows, In the first instance, to qualitatively and quickly assessment what is the current state of the readiness index of the implementation of Industry 4.0 based on success factors that are the result of the experience of countries that already have generated lessons learned in said implementation, and it is easier to focus efforts to lay the foundations of the fourth industrial revolution, identify the current state of the industry to direct public policies, private investment. To identify the current situation of Industry 4.0 in Ecuador, it is proposed to evaluate the readiness index according to the following five success factors: strategy, technology, innovation capacity, innovation ecosystems and manage of change.
\end{abstract}

Keywords: Industry 4.0; strategy; innovation; manage of change

Artículo recibido: 05 de Mayo 2021 Aceptado para publicación: 20 de Junio 2021

Correspondencia: aldowav@gmail.com Conflictos de Interés: Ninguna que declarar 


\section{INTRODUCCIÓN}

Se ha escrito mucha literatura de la base teórica de la industria 4.0 (I4.0) a nivel mundial, pero en países en vías de desarrollo en donde aún la tercera revolución industrial está en marcha se debe tomar como base las lecciones aprendidas por países que llevan la delantera para la implementación de la I4.0. El presente artículo en primera instancia detalla los factores diferenciadores que han sido identificados para la implantación de la industria 4.0 y propone un método ajustado a la realidad de industrias emergentes, en este caso la industria ecuatoriana, que evalúe el punto de partida y tenga herramientas suficientes de manera cualitativa que faciliten su posterior implementación.

Ubicando al Ecuador en contexto industrial, usando como referencias índices relacionados a la I4.0 se tiene: según el Foro Económico Mundial, Ecuador en la evaluación de preparación para la industria "Readiness overall assessment" está ubicado en su ranking mundial en el puesto 89 de 100 según el reporte "Readiness for the Future of Production Report 2018" (World Economic Forum and Kearney 2018), la misma organización para el 2019 ubica al Ecuador en el puesto 90 de 141 de acuerdo con el índice de competitividad considerando el reporte "The Global Competitiveness Report 2019"(Schwab and World Economic Forum - WEF 2019), en cuanto a la gestión de energía para la transición a un sistema energético sostenible, asequible e inclusivo, el Foro Económico Mundial en su reporte "Fostering Effective Energy Transition" en su edición del 2020 ubica a nivel mundial al Ecuador 45 de 115. la Organización Mundial de la Propiedad Intelectual (OMPI) en la publicación “Global Innovation Index 2020” según su índice global ubica al Ecuador en el puesto 99 de 131. (World Intellectual Property Organization 2020), según el reporte “The Network Readiness Index 2020”, que relaciona la conectividad, acceso y la tecnología utilizada para proveer internet posiciona a Ecuador en el puesto 85 de 134 a nivel mundial. En su página web Scimago Journal \& Country Rank, para el 2020, en la cantidad de artículos científicos publicados ubica al Ecuador en el puesto 66 de 234.(SCImago 2021) Considerando al capital humano como un factor clave para el crecimiento, el desarrollo y competitividad en el reporte "The Global Human Capital Report” en su edición del 2017 en su ranking mundial ubica al Ecuador en el puesto 76 de 130 .

Claramente, Ecuador se encuentra en desventaja a nivel mundial, además, el tamaño de las industria ecuatoriana frente a las de los principales países industrializados como lo 
son Japón, Corea y Alemania (World Economic Forum and Kearney 2018) se puede considerar como una industria mediana o pequeña y debe ser evaluada como tal. Según Rauch "es particularmente importante para transformar a las pequeñas y medianas empresas (PYME) en fábricas inteligentes en simbiosis con el desarrollo y cambio de trabajo y los perfiles de cualificación necesarios de las personas trabajando en futuras fábricas inteligentes.(Rauch, Linder, and Dallasega 2020). Además, para la industria ecuatoriana "el principal objetivo incluye la individualización de requerimientos del cliente, flexibilidad y adaptabilidad de manufactura y sistemas logísticos, mejora de la toma de decisiones, integración de tecnologías de información y comunicación y sistemas ciber físicos, la introducción tecnologías avanzadas de producción como lo son: manufactura aditiva, manufactura precisa, etc., conceptos de automatización inteligente, negocios adaptados y modelos organizacionales, así como conceptos para una producción más sustentable y procesos logísticos".(Rauch, Vickery, et al. 2020).

Con una visión más general, Wang concluye que "la idea central de la Industria 4.0 es utilizar tecnologías emergentes de manera que los procesos de negocios y de ingeniería estén profundamente integrados, haciendo que la producción opere de una manera flexible, eficiente y sostenible con una alta calidad y bajo costo constantemente".(Wang et al. 2016). En estudios previos realizados localmente para el sector manufacturero ecuatoriano considerando la aplicación en Ecuador de la I4.0, "se establece que la implementación de tendencias 4.0 apoya al mejoramiento continuo dentro de las empresas manufactureras, cambian así la manera actual de manejar una línea de producción, De todo el análisis se tiene que, los factores más importantes para la implementación de tendencias 4.0 están. 1) La capacidad de inversión en tecnología. 2) Almacenamiento y manejos de datos importantes para la empresa. 3) Nivel de capacitación y actualización de conocimientos de las empresas. 4) Creación y adaptación de nuevos productos.(Banegas Bravo and Arroyo Morocho 2020)

\section{ESTRATEGIAS METODOLÓGICAS}

Para el presente análisis centrado en la I4.0 como factor diferenciador, se consideran cinco áreas prioritarias que representan factores críticos de éxito y has sido facilitadores para la adopción de tecnologías según expertos y empresas manufactureras en el Espacio Alpino. (Biegler et al. 2018) 
El punto de partida para realizar una evaluación cualitativa o describir un modelo de evaluación se debe cubrir con las necesidades de la audiencia destinataria, se reflejan en por qué buscan aplicar el modelo, cómo el modelo puede aplicarse a diferentes estructuras organizativas, quién debe participar en la aplicación del modelo y qué se puede lograr mediante la aplicación del modelo., además, la división de cada punto de evaluación se estructura para que se tenga un criterio de evaluación en escala dividida en varias partes o etapas, el número de etapas puede variar de un modelo a otro, pero lo importante es que en las etapas finales son distintas y bien definidas, y que hay una progresión lógica a través de las etapas.(de Bruin et al. 2005). El procedimiento de evaluación propone factores de éxito inicien con Nivel 0 si no se tiene ningún tipo de aplicación hasta el Nivel 4 si se tiene una implementación completa.

Estrategia - Según, Müller, menciona que "hasta ahora, las pequeñas y medianas empresas suelen considerar a la Industria 4.0 como un medio de mejorar la eficacia, pero no de explorar nuevas oportunidades de negocios. Apoyar a las PYME en la exploración de nuevos modelos de negocio, el estado puede proporcionar información específica, como las mejores prácticas".(Müller, Buliga, and Voigt 2020). Sin una estrategia, la industria ecuatoriana en conjunto no puede enfocarse al cumplimiento de una meta, que debería ser, ponerse a la par de sistemas industrializados a nivel mundial. Se propone identificar el nivel de madurez de la estrategia para la implementación de la I4.0 según la siguiente escala:

- Nivel 0 - la compañía no tiene ninguna estrategia.

- Nivel 1 - la empresa realiza inversiones «forzadas» para probar nuevas tecnologías y / o mejorar el desempeño de sus productos o procesos (sin una estrategia clara y definida)

- Nivel 2 - la empresa realiza inversiones «intencionales» para mejorar el rendimiento de sus productos o procesos.

- Nivel 3 - la empresa tiene una estrategia clara y definida, cuestiona su negocio actual y / o próximo modela e integra tecnología.

- Nivel 4 - la empresa tiene una estrategia 4.0 completa y desarrolla tecnologías dedicadas.

Tecnología - El cambio de tecnología en las anteriores revoluciones industriales es marcado por una mejora significativa en el rendimiento de los procesos y operaciones, en 
la reducción de la energía consumida y en la reducción de la participación del ser humano en operaciones que demanden mucho esfuerzo. Aunque la Industria 4.0 se encuentra actualmente en su etapa de desarrollo para sus infraestructuras de sistema y no hay demasiadas oportunidades para recopilar datos de la vida real para un mayor análisis de datos, eventualmente requiere la conexión perfecta entre las infraestructuras del sistema y el análisis de datos para lograr la autonomía total(Xu and Duan 2019). Considerando que la industria ecuatoriana haya implementado de manera aislada cierto tipo de avances tecnológicos a su producción para la mejora de productos y eficiencia en su sistema productivo se propone medir el nivel de madurez de la aplicación de la tecnología de la I4.0 considerando la siguiente escala:

- Nivel 0 - la empresa no invierte en ninguna tecnología 4.0.

- Nivel 1 - la empresa invierte en tecnología 3.0 para actualizar su sistema de producción.

- Nivel 2 - la empresa prueba o invierte en alguna tecnología 4.0 aislada.

- Nivel 3 - la empresa integra y utiliza tecnología 4.0.

- Nivel 4 - la empresa se anticipa a las nuevas tecnologías e inicia nuevos desarrollos tecnológicos.

Capacidad de innovación - Muchos estudios de innovación organizacional han medida de la innovación por el número de innovaciones adoptadas en un período de tiempo determinado.(Damanpour 1991) La capacidad de innovación de la industria depende del desarrollo interno de los departamentos de investigación y desarrollo I+D para obtener de alguna manera ventaja competitiva, pero dichas mejoras ya sea en productos como en procesos suele partir de la competencia entre empresas, industrias, países, etc. Para los países con industrias emergentes deben considerar la vinculación con universidades, asociación que daría un impulso a los procesos productivos. La medición del nivel de madurez de las industrias esta direccionado a dichas alianzas, a disponer internamente de departamentos que desarrollen, investiguen e innoven:

- Nivel 0 - la empresa no innova.

- Nivel 1 - la empresa tiene una oficina de ingeniería, pero no un departamento de I + D. 
- Nivel 2 - la empresa desarrolla algunos proyectos internos y utiliza recursos internos exclusivamente (se puede colaborar con universidades, centros técnicos y de competencia ocasionalmente).

- Nivel 3 - la empresa cuenta con un departamento de I + D + i y participa en desarrollo de proyectos nacionales colaborativos.

- Nivel 4 - la empresa cuenta con un departamento de I + D y participa en proyectos internacionales colaborativos.

Ecosistemas para la ayuda de innovación - En la sección anterior se menciona que debe existir cierto número de innovaciones en el tiempo para que se pueda hablar de innovación, pero estas mejoras deben además, desarrollarse e un entorno adecuado, si a esto se le suma la competencia entre industrias, se crea un ambiente que propicia el desarrollo, a estos entornos se los llama ecosistemas de innovación, que de acuerdo con la definición de Granstrand, "el conjunto en evolución de actores, actividades y artefactos, y las instituciones y relaciones, incluidas las complementarias y relaciones sustitutivas, que son importantes para el desempeño innovador de un actor o una población de actores.”(Granstrand and Holgersson 2020). Ya que ningún sistema se desarrolla de manera aislada Müller concluye en su artículo que "La capacidad de una empresa para interactuar con otras empresas afecta la medida en que puede acceder y transferir conocimiento, y su valor a otros posibles socios en ecosistemas de creación de valor e innovación.(Müller et al. 2020) aquí, es muy importante la participación del estado conjuntamente con el sector industrial privado. Para la evaluación del nivel de madurez en este apartado se cuantifica la presencia de alianzas que fomenten la innovación.

- Nivel 0 - la empresa no cuenta con ningún apoyo FoF (fabrica del futuro.)

- Nivel 1 - la empresa colabora con actores «aislados» y heterogéneos y se beneficia de programas de apoyo general.

- Nivel 2 - la empresa forma parte de redes tecnológicas especializadas y se beneficia de programas de apoyo.

- Nivel 3 - la empresa forma parte de ecosistemas multiactores (clústeres, plataformas...) y se beneficia de programas de apoyo complementarios, originales y de incentivos. 
- Nivel 4 - la empresa forma parte de multiactores estructurados (regionales, nacionales o europeos) ecosistemas y beneficios de políticas públicas y programas de apoyo especializados.

Habilidad y manejo del cambio - De acuerdo con Albach, "los agentes de cambio son necesarios para que una organización cambie con éxito, mientras que su resistencia (y la de otros) puede, por el contrario, condenar todas las iniciativas de cambio al fracaso. Han descubierto que alrededor del $70 \%$ de todas las iniciativas de cambio fracasan debido a la resistencia individual".(Albach et al. 2015) Las industrias emergentes tienen un problema muy visible debido a la cantidad de recursos económicos disponibles para ponerse a la par de industrias europeas o asiáticas, pero existe un factor asociado a la zona de confort en la que se encuentran, ya que con las instalaciones existentes tienen cierto nivel de producción, sin plantear estrategias, sin implementar departamentos de mejora continua, sin comprar o transferir tecnología para incentivar la innovación. El cambio y su manejo debe ser una política interna de cualquier empresa productiva, en la cual el nivel de madurez se mide en el nivel de planificación para abordar el cambio.

- Nivel 0 - El problema no se aborda.

- Nivel 1 - No hay una persona identificada a cargo de la transformación digital, la empresa aborda el problema después de la implementación de las tecnologías.

- Nivel 2 - La empresa evalúa las habilidades internas cuando se implementa la tecnología.

- Nivel 3 - La transformación digital es gestionada por una persona identificada de la dirección, la empresa diseña un plan antes de la implementación de las tecnologías.

- Nivel 4 - La empresa implementa un Planificación de Recursos Humanos (PRH), hay una nueva cultura y mente puesta en la empresa.

\section{RESULTADOS}

Debido, a la falta de políticas públicas que incentiven la implementación de la I4.0 en Ecuador se ve la necesidad de crear una ruta de trabajo que inicie con la evaluación cualitativa para luego con los resultados plantear un espacio de trabajo, incentivar la creación de ecosistemas de innovación y de una manera obligar que la desventaja que actualmente se tiene frente al desarrollo a nivel mundial medir el nivel de implementación o de madurez de la I4.0 como factor diferenciador en la industria ecuatoriana. 
La evaluación cualitativa propuesta brinda una visión rápida del nivel de madurez de los factores de éxito: estrategia, tecnología, capacidad de innovación, ecosistemas de ayuda para la innovación y habilidades y manejo del cambio. Para luego, mediante una comparativa más profunda con niveles de madurez de países industrializados crear una ruta de trabajo.

\section{DISCUSIÓN}

Los factores de éxito que han sido las claves para dar el salto a la I4.0 y a la vez que son lecciones aprendidas por países industrializados nos dan una pauta para dar el paso inicial enfocados en la aplicación de acuerdo con el tipo de industria disponible, que según el Instituto Nacional de Estadística y Censos (INEC) en su boletín técnico de enero 2020, lista las principales industrias ecuatorianas de acuerdo con el Índice de Producción de la Industria Manufacturera (IPI-M), donde, la industria de los productos alimenticios, bebidas y tabaco; textiles, prendas de vestir y productos de cuero tiene una cuota del 49,40\%. Considerando que, Ecuador tiene la mitad de su producción industrial asociada al agro e industria de alimentos, de acuerdo con el estudio presentando por Djatna, que vincula el sector agroindustrial, el pensum académico de las universidades y la I4.0, menciona que "es urgente repensar y redefinir cómo preparar una nueva perspectiva en los tres disciplinas combinadas que forman la ingeniería industrial y de sistemas, ingeniería de procesos / bioprocesos e ingeniería ambiental industrial" asociando a lo antes mencionado, la industria ecuatoriana además, deberá mejorar la capacidad de generar tecnología, fomentar la transferencia de conocimiento, implementar infraestructura de la industria inteligente y mediante la circulación universidad-industria crear ecosistemas que faciliten la capacidad de innovación.

\section{LISTA DE REFERENCIAS}

Albach, Horst, Heribert Meffert, Andreas Pinkwart, and Reichwald Ralf. 2015. Management of Permanent Change-New Challenges and Opportunities for Change Management.

Banegas Bravo, Jennifer Dayanna, and Flavio Roberto Arroyo Morocho. 2020. "Estudio de Las Tendencias 4.0 y Su Relación Con El Diseño Industrial Para El Desarrollo

Del Sector Industrial Manufacturero de La Zona 9 Del Ecuador.” Universidad Central del Ecuador.

Biegler, Christoph, Wilfried Sihn, Arko Steinwender, Valerie Rocchi, and Alessandro 
Sala. 2018. "Adoption of Factory of the Future Technologies Concept of an Impact Indicator System to Track the Path towards the Factory of the Future." 2018 Ieee International Conference on Engineering, Technology and Innovation (Ice/Itmc).

de Bruin, Tonia, Michael Rosemann, Ronald Freeze, and Uday Kulkarni. 2005. "Understanding the Main Phases of Developing a Maturity Assessment Model." ACIS 2005 Proceedings - 16th Australasian Conference on Information Systems (January).

Damanpour, FARIBORZ. 1991. "Meta_Leadership.Pdf." Academy of Management Journal 34(3):555-90.

Granstrand, Ove, and Marcus Holgersson. 2020. "Innovation Ecosystems: A Conceptual Review and a New Definition." Technovation 90-91(May). doi: 10.1016/j.technovation.2019.102098.

Müller, Julian M., Oana Buliga, and Kai Ingo Voigt. 2020. "The Role of Absorptive Capacity and Innovation Strategy in the Design of Industry 4.0 Business ModelsA Comparison between SMEs and Large Enterprises." European Management Journal (xxxx):1-11. doi: 10.1016/j.emj.2020.01.002.

Rauch, Erwin, Christian Linder, and Patrick Dallasega. 2020. “Anthropocentric Perspective of Production before and within Industry 4.0." Computers and Industrial Engineering 139(xxxx):105644. doi: 10.1016/j.cie.2019.01.018.

Rauch, Erwin, Andrew R. Vickery, Christopher A. Brown, and Dominik T. Matt. 2020. SME Requirements and Guidelines for the Design of Smart and Highly Adaptable Manufacturing Systems.

Schwab, Klaus, and World Economic Forum - WEF. 2019. Insight Report - World Economic Forum.

SCImago. 2021. "NoScimago Journal \& Country Rank." Retrieved (https://www.scimagojr.com/countryrank.php?year=2020).

Wang, Shiyong, Jiafu Wan, Di Li, and Chunhua Zhang. 2016. "Implementing Smart Factory of Industrie 4.0: An Outlook." International Journal of Distributed Sensor Networks 2016. doi: 10.1155/2016/3159805.

World Economic Forum, and A. T. Kearney. 2018. Insight Report: Readiness for the Future of Production Report 2018. 
World Intellectual Property Organization, WIPO. 2020. Who Will Finance Innovation? Global Innovation Index. edited by S. W.-V. Soumitra Dutta, Bruno Lanvin.

Xu, Li Da, and Lian Duan. 2019. "Big Data for Cyber Physical Systems in Industry 4.0: A Survey." Enterprise Information Systems 13(2):148-69. doi: 10.1080/17517575.2018.1442934. 